\title{
Excitation Energy Migration Processes in Cyclic Porphyrin Arrays Probed by Single Molecule Spectroscopy
}

Jaesung Yang ${ }^{\dagger}$, Mira Park ${ }^{\dagger}$,Zin Seok Yoon ${ }^{\dagger}$, Takaaki Hori ${ }^{+}$, Xiaobin Peng ${ }^{\dot{t}}$, Naoki Aratani ${ }^{+}$, Peter Dedecker ${ }^{\S}$, Jun-ichi Hotta ${ }^{\S}$, Hiroshi Uji-i ${ }^{\S}$, Michel Sliwa ${ }^{\S}$, Johan Hofkens ${ }^{*}$, Atsuhiro Osuka ${ }^{* \neq}$ and Dongho Kim ${ }^{* \dagger}$

${ }^{\dagger}$ Center for Ultrafast Optical Characteristics Control and Department of Chemistry, Yonsei University, Seoul 120-749, Korea, ${ }^{\dagger}$ Department of Chemistry, Graduate School of Science, Kyoto University, Sakyo-ku, Kyoto 606-8502, Japan, ${ }^{\S}$ Department of Chemistry, Katholieke Universiteit Leuven and Institute for Nanoscale Physics and Chemistry (INPAC), Celestijnenlaan 200F, 3001 Heverlee, Belgium.

E-mail: johan.hofkens@,chem.kuleuven.ac.be, osuka@kuchem.kyoto-u.ac.jp, dongho@yonsei.ac.kr 


\section{Supporting Information 1.}

(a)

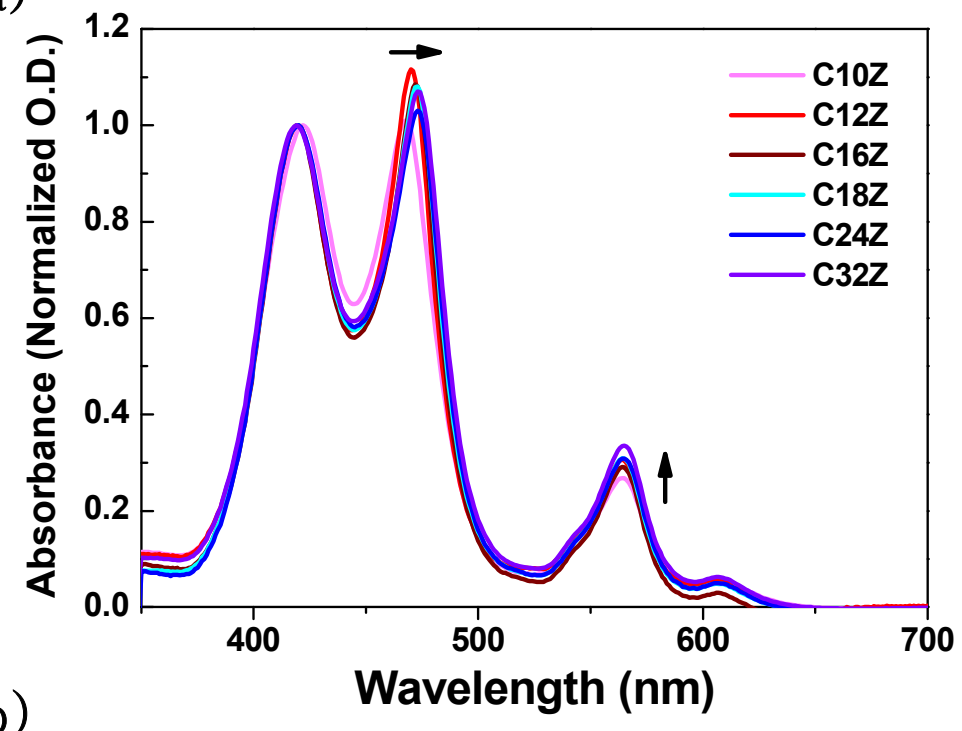

(b)

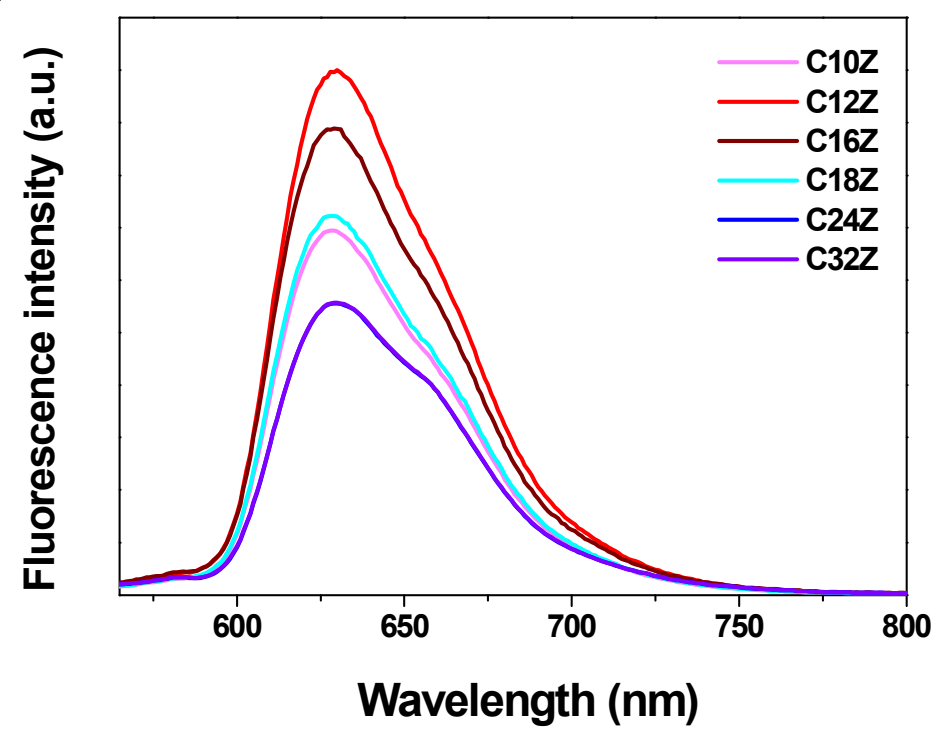

Figure S1. Steady-state absorption and emission spectra of the cyclic porphyrin arrays from $\mathbf{C 1 0 Z}$ to $\mathbf{C 3 2 Z}$ in toluene. In the UV-visible absorption spectra, the low-energy Soret band locates nearly at the same position as that of porphyrin monomer Zn(II)TPP. The Soret band splitting in the cyclic porphyrin arrays, according to the dipole-dipole coupling between porphyrin dimer subunits via the 1,3-phenylene spacer increases slightly as the number of dimer subunits increases. On the other hand, the band shifts in the Q-bands are not so significant and the Q-bands show a gradual increase in their intensities (a). The fluorescence spectra were obtained with the excitation wavelength at $550 \mathrm{~nm}$ (b). On the basis of absorption and emission behavior, the result indicates that nonradiative quenching sites increase as the cyclic porphyrin arrays become larger. 


\section{Supporting Information 2.}
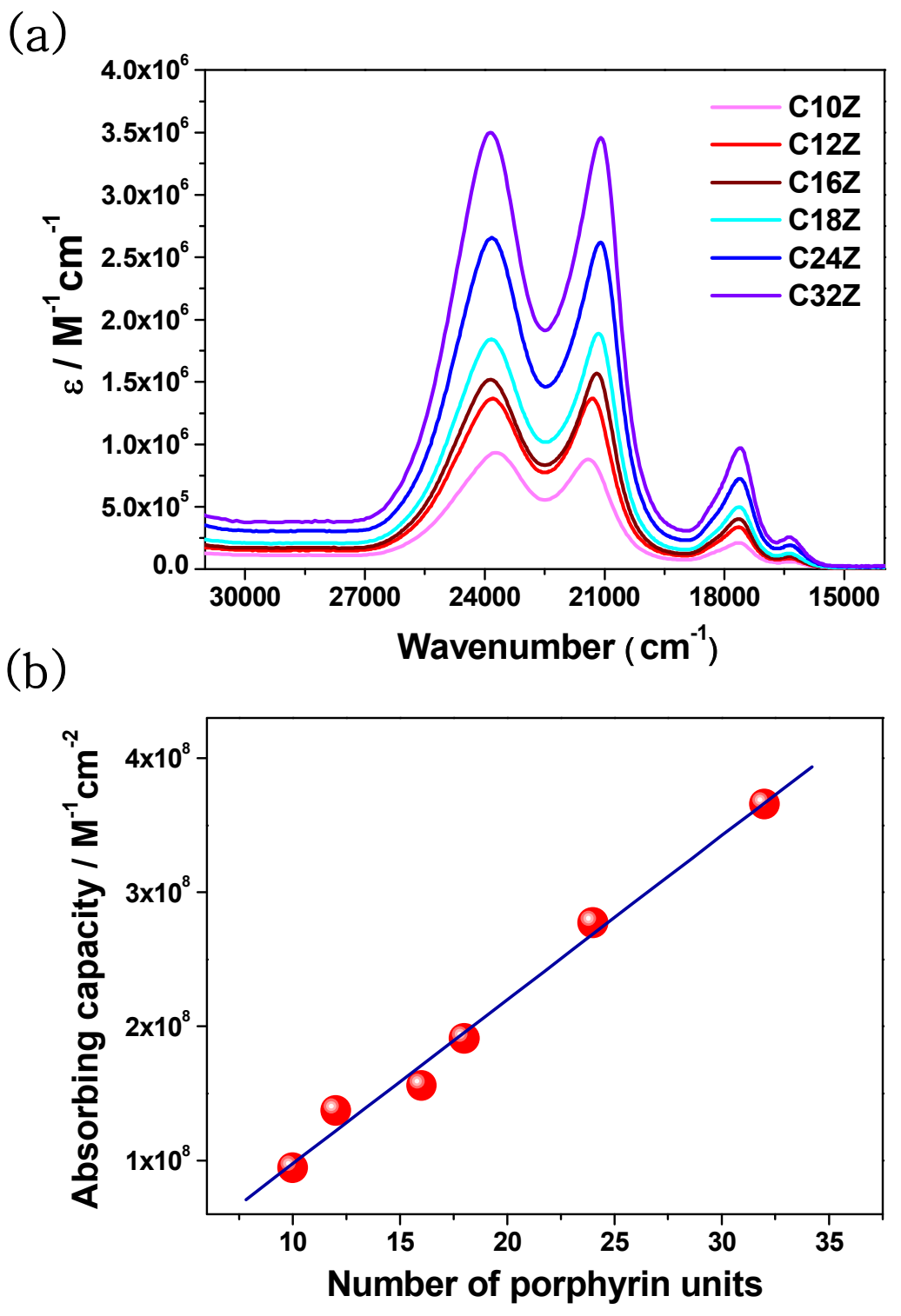

Figure S2. Molar extinction coefficient spectra of the cyclic porphyrin arrays from $\mathbf{C 1 0 Z}$ to $\mathbf{C 3 2 Z}$ in toluene (a) and the plot of the absorbing capacity as a function of the number of porphyrin units in the arrays (b). The values of the absorbing capacities are evaluated by integrating the molar extinction coefficient spectrum for each cyclic porphyrin arrays. 


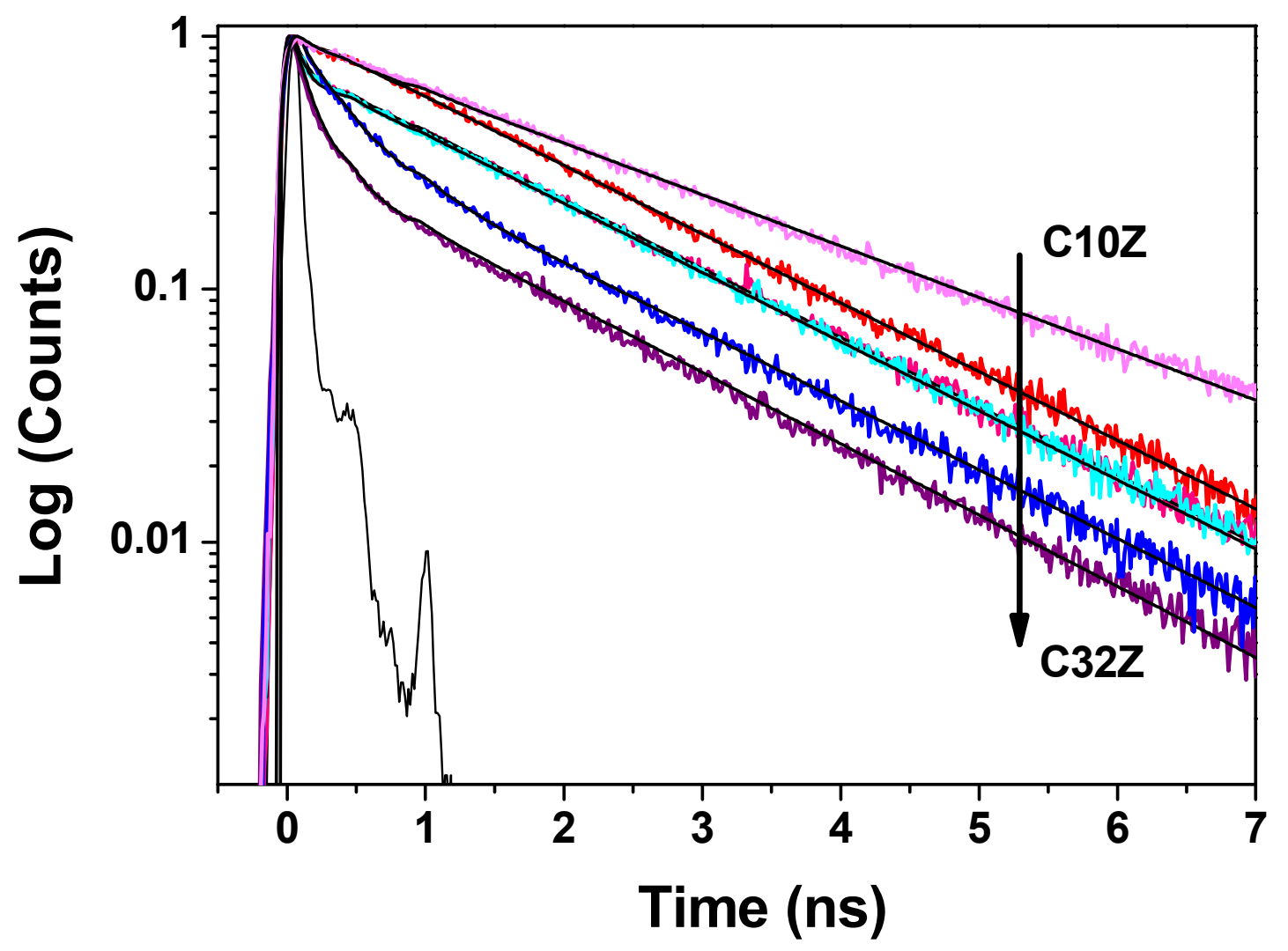

Figure S3. Fluorescence decay profiles of the cyclic porphyrin arrays from $\mathbf{C 1 0 Z}$ to $\mathbf{C 3 2 Z}$ in toluene. The excitation wavelength of $400 \mathrm{~nm}$ and detection wavelength of $625 \mathrm{~nm}$ were used. As the cyclic porphyrin array becomes larger, the average fluorescence lifetime decreases up to the largest cyclic porphyrin array $\mathbf{C 3 2 Z}$. In relatively small cyclic porphyrin arrays $\mathbf{C 1 0 Z}$ and $\mathbf{C 1 2 Z}$, the fluorescence lifetimes show single exponential decay. On the other hand, in larger cyclic porphyrin arrays than $\mathbf{C 1 2 Z}$, the fluorescence temporal profiles start to show double exponential decay. The contribution by the fast decay component in double exponential decay increases as the cyclic porphyrin arrays become larger. From these spectral data, such a feature is believed to arise from the increase of nonradiative decay channels due to the formation of flexible structures in larger cyclic porphyrin arrays. 


\section{Supporting Information 4.}

(a)
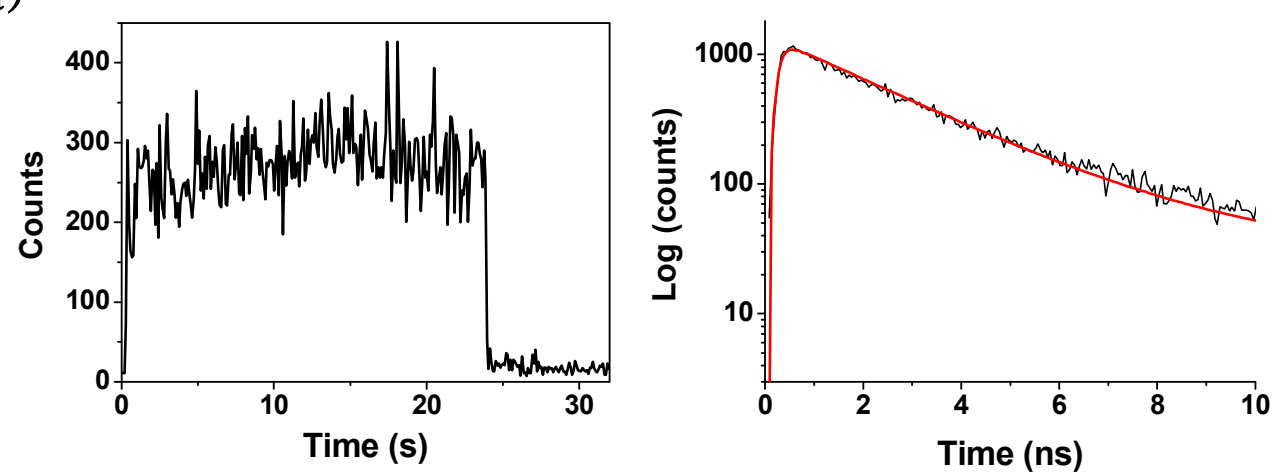

(b)
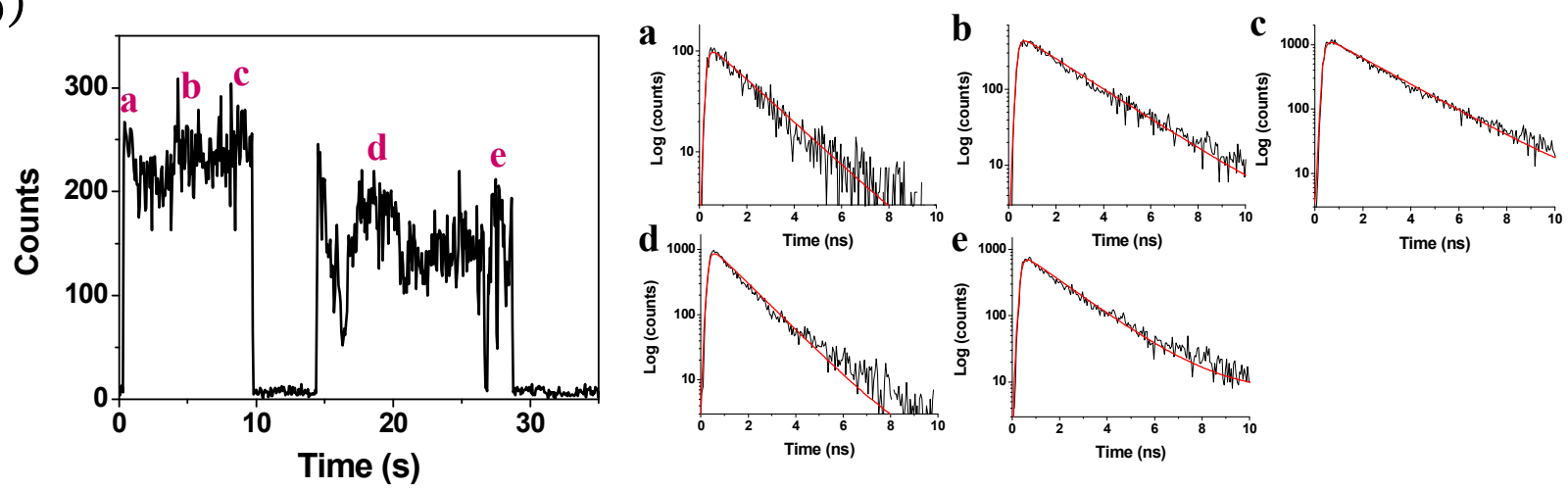

Figure S4. Representative fluorescence intensity trajectories (FITs) with $50 \mathrm{~ms}$ binning time and fluorescence lifetimes showing different behaviors of single C12Z molecules: Single-step photobleaching in FITs and the corresponding fluorescence decay (2.2 ns) (a). Stepwise photobleaching: the fluorescence lifetimes are a; 1.65, b; 1.98, c; 2.04, d;1.19, e;1.83 ns (b). 